\title{
3 Research Square

\section{Standardized Reporting and Quantification of Whole-Body MRI findings in Children with Chronic Non-Bacterial Osteomyelitis Treated with Pamidronate}

Caroline Marie Andreasen ( $\square$ caroline.marie.andreasen@rsyd.dk)

Vejle Hospital: Sygehus Lillebalt Vejle Sygehus https://orcid.org/0000-0002-2376-0446

Rikke Fuglsang Klicman

Aarhus University Hospital Skejby: Aarhus Universitetshospital

Troels Herlin

Aarhus University Hospital Skejby: Aarhus Universitetshospital

Ellen Margrethe Hauge

Aarhus University Hospital Skejby: Aarhus Universitetshospital

Anne Grethe Jurik

Aarhus University Hospital Skejby: Aarhus Universitetshospital

\section{Research article}

Keywords: Chronic non-bacterial osteomyelitis, chronic recurrent multifocal osteomyelitis, case-study, whole-body MRI, diphosphonates, pamidronate, standardized reporting

Posted Date: February 18th, 2022

DOl: https://doi.org/10.21203/rs.3.rs-1251361/v1

License: (c) (i) This work is licensed under a Creative Commons Attribution 4.0 International License.

Read Full License 


\section{Abstract}

\section{Objectives}

To test a modified radiological index for non-bacterial osteitis (mRINBO) used for standardized reporting and quantification of whole-body MRI (WBMRI) findings in children with chronic non-bacterial osteomyelitis (CNO) receiving pamidronate therapy.

\section{Methods}

WBMRI was assessed in 18 children with $\mathrm{CNO}$ at baseline and after receiving pamidronate therapy for one year. Parameters of interest were: number and anatomic site of radiologically active bone lesions (RAL), size of RAL, extramedullary affection, spinal involvement and changes in mRINBO, which includes both the number and maximal size of RAL (RALmax) in addition to extramedullary and chronic changes.

\section{Results}

At the time of diagnosis, the mean age of the children was 9.8 (sd, 8.7-10.9) years and 11/18 were females. The number of RALs per patient decreased from median [interquartile range] 4.5 [3-8] to 3 [2-5] RALs per patient $(p=0.02)$ and extramedullary inflammatory changes regressed. Sixty-one percent of all RALs occurring at baseline resolved and three children became without active inflammatory lesions by WBMRI. However, the median size of RALs did not change when taking new lesions occurring in 7/18 children into account, but RALmax decreased significantly from 39 [29-45] $\mathrm{mm}$ at baseline to 28 [20-40] $\mathrm{mm}(p<0.01)$ at year-one with a concomitant decrease of mRINBO from a median of 5 [4-7] to 4 [3-5] $(p=0.05)$.

\section{Conclusions}

mRINBO decreased during pamidronate therapy and mRINBO may be a potential scoring method to quantify changes in radiological disease activity in children with CNO. Further studies are needed to test feasibility and validity of mRINBO.

\section{Introduction}

Chronic non-bacterial osteomyelitis (CNO) is a skeletal disorder in children and adolescents. CNO is characterized by sterile and often multifocal inflammatory bone lesions.[1] Bone lesions have a predilection for the metaphyses of the long tubular bones in the lower extremities, the axial skeleton and the clavicles. During the disease course bone lesions can vanish or persist and bone lesions may occur at new sites.[2-6] Children with CNO present clinically with musculoskeletal complaints such as pain and swelling at the skeletal sites involved and spinal lesions can lead to vertebral fractures and risk of kyphoscoliosis.[7, 8] However, lesions may also be asymptomatic.[2, 3, 9] 
Active inflammation in bone, joints and soft tissue can be detected by whole-body MRI (WBMRI) [10, 11], which is often used in clinical practice to diagnose CNO.[12] WBMRI is useful to detect multifocal bone lesions at typical locations initially and at any follow-up examination. $[5,12,13]$ WBMRI can also be used to detect complications, new lesions and clinically silent lesions when monitoring the disease during treatment.[2,14] In spondyloarthritis and in adults with CNO standardized radiological outcome measures can be used to assess changes in radiological disease activity.[15-19] However, in children with CNO, a standardized radiological outcome measure is needed to assess radiological disease burden as well as changes in radiological disease activity following treatment.[20]

A radiological index for non-bacterial osteitis (RINBO) has been suggested for standardized reporting of WBMRI in patients with CNO.(21) RINBO includes four parameters of interest: number and maximal size of radiological active lesions, extramedullary changes and spine involvement encompassing both active and chronic lesions in the form of osseous hyperostosis and vertebral deformity (Table 1). A RINBO scoring is easy to perform and results in a summary regarding disease severity. However, it has not been evaluated in longitudinal studies. A more complicated scoring tool has been evaluated in a longitudinal therapeutic study, but primarily with regard to observer variation by assessment of the various CNO lesions with no concomitant summation of disease severity at patient level.[22, 23] 
Table 1

The original and the modified radiologic index for standardized reporting of whole-body MRI in patients with chronic non-bacterial osteomyelitis
Parameter of interest
Criterion RINBO
Criterion mRINBO
Value

No of patient's radiological active lesions

$\begin{array}{llcl}\text { Unifocal } & 1 & 1 & 1 \\ \text { Paucifocal } & 2-4 & 2-4 & 2 \\ \text { Multifocal } & \geq 5 & \geq 5 & 3\end{array}$

Maximum size of patients' radiological active lesion

$\begin{array}{lllr}\text { Minor } & <10 \mathrm{~mm} & <20 \mathrm{~mm} & 1 \\ \text { Average } & 10-100 \mathrm{~mm} & 20-50 \mathrm{~mm} & 2 \\ \text { Major } & >100 \mathrm{~mm} & >50 \mathrm{~mm} & 3\end{array}$

Extramedullary affection

$\begin{array}{lllr}\text { Acute } & \begin{array}{l}\text { Periostal reaction and/or soft } \\ \text { tissue edema }\end{array} & \begin{array}{l}\text { Periostal reaction and/or soft } \\ \text { tissue edema }\end{array} & 1 \\ \text { Chronic } & \text { Hyperostosis } & \text { Hyperostosis } & 1\end{array}$

Spinal involvement

$\begin{array}{lllr}\text { Acute } & \begin{array}{l}\text { Radiologically active vertebral } \\ \text { lesion }\end{array} & \begin{array}{l}\text { Radiologically active vertebral } \\ \text { lesion }\end{array} & 1 \\ \text { Chronic } & \begin{array}{l}\text { CNO related vertebral body } \\ \text { deformation }\end{array} & \begin{array}{l}\text { CNO related vertebral body } \\ \text { deformation }\end{array} & 1\end{array}$

\section{Maximum index point}

RINBO: Radiologic index for chronic non-bacterial osteomyelitis (21)

mRINBO: Modified RINBO score with new definitions of the maximum size of patients' radiological active lesion

based on the current sizes and exclusion of radiological active lesions in the metatarsal bones.

Treatment of CNO is largely empirical. Non-steroidal anti-inflammatory drugs (NSAIDs) are recommended as first-line therapy. $[3,24]$ Children with multiple lesions or spinal involvement are considered to have more challenging disease courses,[6,25] necessitating treatment with second-line pharmaceuticals if NSAIDs fails.[6, 26] The amino bisphosphonate pamidronate has in cases-series been reported to be effective in improving clinical and radiological disease activity.[6, 8, 33-35, 19, 23, 27-32]

The objective of this study was to test a modified radiological index for non-bacterial osteitis (mRINBO) to monitor $\mathrm{CNO}$ in children treated with pamidronate. The study also highlights some of the challenges 
when assessing the radiological disease activity in CNO.

\section{Methods}

\section{Patient selection}

The study cohort consisted of 18 children, representing a subgroup of 51 children previously published [6], having CNO with multifocal or spinal bone inflammation not responding to NSAID treatment and therefore being subjects to second-line pharmaceuticals in the form of intravenous pamidronate for one year. They were all seen in the Department of Pediatrics and Adolescent Medicine, (Institute blinded for review), Denmark, between 2007 and 2015 and were diagnosed with CNO according to the Bristol Criteria. [36] Children were only included if a WBMRI was performed before initiation of pamidronate and following 9-18 months treatment. Children that were concomitantly treated with biological disease modifying anti-rheumatic drugs were excluded.

The following clinical data were collected from the electronic medical records: Age, sex, time from first symptom to diagnosis, time from diagnosis to initiation of pamidronate treatment, comorbidities, concomitant medication, laboratory screening (erythrocyte sedimentation rate (ESR), HLA-B27 status) and performance of bone biopsy.

\section{Clinical assessment}

From clinical records clinical disease activity was assessed at baseline and at the visit closest to WBMRI year-one. Symptoms were defined as patient- or parent-reported musculoskeletal pain and/or observed swelling at the skeletal sites involved. Signs of inflammation were defined as physician-reported swelling or warmth at affected skeletal site, and elevated ESR $(>20 \mathrm{~mm} / \mathrm{h})$ attributable to CNO was used as a biochemical marker of inflammation. Clinical inactive disease was defined as no symptoms or signs of inflammation and a normal ESR for six consecutive months. [6]

\section{WBMRI protocol}

WBMRI was performed at baseline and following one year of pamidronate treatment as part of clinical routine using a 1.5 Tesla Siemens Magnetom Avanto (Erlangen, Germany) or a Philips Achieva dStream (Eindhoven, The Nederland). A coronal whole-body short tau inversion recovery (STIR) sequence with a slice thickness of $5 \mathrm{~mm}$ was performed in all patients and a coronal T1-weighted sequence was performed in 11 children at baseline and in 15 children at year-one. Site specific MRI was performed when clinically indicated, at baseline encompassing sagittal STIR of the spine (8 children), axial STIR of the pelvis ( 8 children), sagittal STIR of the feet ( 6 children) and other anatomical regions ( 6 children) and at year-one sagittal STIR of the spine (6 children), axial STIR of the pelvis (10 children), sagittal STIR of the feet (13 children) and others anatomical regions ( 6 children).

\section{WBMRI assessment}


The WBMRI examinations were retrospectively evaluated on a dedicated PACS workstation (Agfa Impax, Belgium, version 6.3.1.8000) with $2 \mathrm{~K}$ Bracco screens independently by two experienced musculoskeletal radiologists (blinded for review) for the presence and size of any bone lesions. All size measurements were performed on the WBMRI sequences. The radiologists were blinded to clinical data, but due to the metabolic effects of pamidronate at the metaphyses it was not possible to blind assessors to pre-and post-treatment imaging.

At year-one bisphosphonate induced sclerotic and edematous metaphyseal bands parallel to the growth plates could challenge the assessment of bone lesions, especially in the knee region. This was handled with appropriate window setting (Fig. 2).

\section{Anatomical distribution of radiologically active bone lesions}

The location of bone lesions was divided into seven anatomical regions as shown in Table 2. Besides, the location of tubular bone lesions was assessed in relation to epiphysis, metaphysis, diaphysis and hypervascularized growth areas adjacent to apophyses.[37-39] 
Table 2

Distribution of radiologically active bone lesions at baseline and after one year of pamidronate therapy

\begin{tabular}{|c|c|c|c|c|c|}
\hline & Baseline & Year 1 & Baseline & Year 1 & $p$-value \\
\hline & RAL n (\%) & RAL n (\%) & Children n (\%) & Children n (\%) & \\
\hline Head & $1(1)$ & $1(2)$ & $1(6)$ & $1(6)$ & - \\
\hline Mandibula & $1(1)$ & $1(2)$ & $1(6)$ & $1(6)$ & \\
\hline Chest & $6(5)$ & $5(8)$ & $6(33)$ & $5(28)$ & 0.5 \\
\hline Clavicula & $3(3)$ & $3(5)$ & $3(17)$ & $3(17)$ & \\
\hline Sternum & $1(1)$ & 0 & $1(6)$ & 0 & \\
\hline Scapula* & $2(2)$ & $2(3)$ & $2(11)$ & $2(11)$ & \\
\hline Upper extremities & $4(4)$ & 0 & $3(17)$ & 0 & 0.13 \\
\hline Proximal humerus & $1(1)$ & 0 & $1(6)$ & 0 & \\
\hline Distal humerus & $1(1)$ & 0 & $1(6)$ & 0 & \\
\hline Proximal radius/ulna & $1(1)$ & 0 & $1(6)$ & 0 & \\
\hline Distal radius/ulna & $1(1)$ & 0 & $1(6)$ & 0 & \\
\hline Pelvis & $21(19)$ & $13(21)$ & $10(56)$ & $8(44)$ & 0.06 \\
\hline Pelvis-ileum* & $11(10)$ & $5(8)$ & $8(44)$ & $4(22)$ & \\
\hline Pelvis-pubis & $1(1)$ & $1(2)$ & $1(6)$ & $1(6)$ & \\
\hline Pelvis-ischium & $2(2)$ & $3(5)$ & $2(11)$ & $3(17)$ & \\
\hline Pelvis-sacrum & $7(6)$ & $4(7)$ & $6(33)$ & $4(22)$ & \\
\hline Distal extremities & $49(45)$ & $26(43)$ & $16(89)$ & $9(50)$ & $<0.01$ \\
\hline Proximal femur* & $10(9)$ & $6(10)$ & $7(39)$ & $3(17)$ & \\
\hline Distal femur & $8(7)$ & $3(5)$ & $6(33)$ & $2(11)$ & \\
\hline Proximal tibia* & $11(10)$ & $4(7)$ & $9(50)$ & $3(17)$ & \\
\hline Distal tibia* & $15(14)$ & $9(15)$ & $9(50)$ & $6(33)$ & \\
\hline Proximal fibula & 0 & 0 & 0 & 0 & \\
\hline Distal fibula & $5(4)$ & $4(7)$ & $4(22)$ & $3(17)$ & \\
\hline Feet & $13(12)$ & $10(17)$ & $8(44)$ & $6(33)$ & 0.55 \\
\hline Foot-calcaneus * & $5(4)$ & $6(10)$ & $4(22)$ & $4(22)$ & \\
\hline
\end{tabular}




\begin{tabular}{|c|c|c|c|c|c|}
\hline & Baseline & Year 1 & Baseline & Year 1 & $p$-value \\
\hline Foot- talus* & $3(3)$ & $2(3)$ & $3(17)$ & $2(11)$ & \\
\hline Foot-middle & $5(4)$ & $2(3)$ & $4(22)$ & $1(6)$ & \\
\hline Spine & $16(15)$ & $6(10)$ & $6(33)$ & $3(17)$ & $<0.01$ \\
\hline Cervical vertebra & 0 & 0 & 0 & 0 & \\
\hline Thoracic vertebra* & $15(14)$ & $2(3)$ & $5(28)$ & $2(11)$ & \\
\hline Lumbar vertebra* & $1(1)$ & $4(7)$ & $1(6)$ & $1(6)$ & \\
\hline Total & $110(100)$ & $61(100)$ & - & - & \\
\hline \multicolumn{6}{|c|}{$\begin{array}{l}\text { Distribution of radiologically active lesions (RALs) at baseline and after one year of pamidronate } \\
\text { treatment ( } n=18 \text { ). Anatomic locations were categorized into seven anatomic regions. Anatomic } \\
\text { distribution of RALs was assessed by WBMRI prior to and following one year of pamidronate } \\
\text { treatment. RALs are presented as number (and percentage of total RALs) prior to pamidronate (total } \\
110 \text { RAL) and year one (total } 61 \text { RAL). RALs are also presented as number of children (and } \\
\text { percentage of } 18 \text { children) with RALs at the different anatomic locations. McNemar's test was used } \\
\text { to compare distribution of RALs in children during pamidronate treatment. P-value }<0.05 \text { was } \\
\text { considered significant. *Indicates anatomic locations with new RAL at year one. }\end{array}$} \\
\hline
\end{tabular}

\section{Definitions of parameters of interest}

Radiologically active lesions (RALs) were defined as areas of increased signal intensity in the bone marrow on STIR images, and concomitant periosteal edema and/or soft tissue edema was defined as active extramedullary changes. Cortical thickening or bone-expansion were defined as chronic hyperostotic changes and deformation of vertebra was interpreted as sign of chronic spinal involvement. The size of all RALs and the size of the largest RAL for each patient (RALmax) were recorded, and the height of affected vertebral bodies was measured.

\section{Modified radiological index for non-bacterial osteitis (mRINBO)}

A composite quantification of the above stated parameters assessed by WBMRI was elaborated in accordance with the RINBO method (Table 1).[21] Due to the size of RAL measured in the current material at baseline and year-one a modification of the RINBO was found to be needed with a reduced size of RALmax, resulting in a modified ( $m$ )RINBO quantification used for all WBMRI (Table 1). Also, the metatarsal bones were excluded from the assessment due to a general poor visualization on coronal images, and known difficulties regarding differentiation of metatarsal CNO lesions from non-specific hyperintensities in the feet.[40]

\section{Statistical analysis}

Normally distributed data were presented as mean (standard deviation, sd) and data not normally distributed were presented as median [interquartile range, IQR]. Comparison between baseline and year 
one was made using Wilcoxon signed rank test for categorical data and McNemars test for paired dichotomous data. A bootstrapping method was used to determine changes in the size of all RALs. Pvalues $<0.05$ were considered statistically significant. The statistical analyses were performed using Stata 14 (StataCorp).

\section{Results}

\section{Patients' characteristics}

Fifty-one children were diagnosed with CNO during the period 2007-2015 [6] and 18 children fulfilled the study criteria (Fig. 1). At time of diagnosis the mean age of the children was 9.8 (sd, 8.7-10.9) years and $11 / 18$ children were females. The time from first symptoms to diagnosis was median 11 [IQR, 5-24] months. All children were treated with NSAIDs initially, but none of them received conventional DMARDs or glucocorticoids. Pamidronate treatment was initiated (baseline) median 5 [IQR, 0.7-9.2] months after the diagnosis was obtained. The first WBMRI was performed mean 1.2 (sd, 0.7-1.7) months prior to initiation of pamidronate treatment and follow-up WBMRI was performed after a mean of 12 (sd, 11.113.3) months.

From baseline to year-one ESR decreased from median 14 [IQR, 9-23] $\mathrm{mm} / \mathrm{h}$ to 7 [IQR, 3-12] $\mathrm{mm} / \mathrm{h}$ $(p=0.01)$. One of 9 children tested was HLA-B27 positive. A bone biopsy was performed in 13/18 children, revealing only non-specific inflammatory changes.

\section{Anatomical distribution of radiologically active bone lesions:}

The most common locations of RALs were in the long tubular bones of the lower extremities, pelvis and spine (Table 2). The majority of RALs in the tubular bones and the pelvis were located to hypervascularized areas adjacent to growth zones such as metaphyseal cartilage plates and cartilage at apophyses, especially at the trochanter region. Thus, at baseline 5 of 10 RALs in the proximal femur were located to the apophyseal region at the trochanter and five at the growth plate. Most of the 21 pelvic RALs were also located adjacent to the growth areas; five occurred at the triangular cartilage, two at the ischiopubic synchondrosis, six at the acetabular roof, seven in the sacrum adjacent to the SI-joint and one in the ischium. (Table 2, Fig. 2).

\section{Number of radiological active bone lesions}

At baseline, the total number of RALs was 110 with a median of 4.5 [IQR, 3-8] RALs per patient. The RALs were symmetrically located in 17/18 children. From baseline to year-one, 67/110 (61\%) RALs resolved, and $3 / 18$ children became without signs of radiological activity by WBMRI (Fig. 3). However, 18 new RALs appeared in 7/18 (39\%) of the children at year-one. The total number of RALs at year-one was 61 with a median of 3 [IQR, 2-5] RALs per patient, which was a significant decrease of the total number of RALs per patient from baseline to year-one $(p=0.02)$ (Table 2, Fig. 3 ). 
Spinal involvement encompassed 16 vertebral RALs in 6/18 (33\%) of the children at baseline. At year-one $14 / 16$ vertebral lesions had resolved ( $<<0.01$ ) (Fig. 3), but one child without vertebral lesions at baseline developed four new asymptomatic lumbar vertebral RALs (Table 2, Fig. 3 and supplementary file S1). Vertebral deformation was observed in 4 children at baseline with no improvement during pamidronate therapy.

\section{Size of radiological active lesions}

At baseline, the median size of RALs ( $\mathrm{n}=110)$ was 21 [IQR, 14-29] mm which decreased to 0 [IQR, 0-22] $\mathrm{mm}$ at year-one, $\mathrm{p}<0.01$ (Supplementary file S2). However, due to the new RALs developing from baseline to year-one the median size of all RALs at year-one $(n=61)$ was 22 [IQR, 5-31] mm and thus did not change from baseline. However, the median size of RALmax decreased from 39 [IQR, 26-45] mm at baseline to 28 [IQR, 20-40] mm at year-one $(p<0.01)$ (Fig. 3). The largest RALmax at baseline was $65 \mathrm{~mm}$ and at year-one $78 \mathrm{~mm}$, both were symptomatic and located at the pelvic triangular cartilage.

\section{Extramedullary affection}

At baseline, extramedullary active inflammatory changes were observed in relation to 9 of the $110(8 \%)$ RALs in 7 children, being located to the mandible, clavicle, pelvic bones or long tubular bones in the lower extremities. Except in one patient, all the extramedullary edematous changes resolved during the study period (Fig. 2). New extramedullary changes were not observed from baseline to year one. Hyperostosis was observed in relation to the clavicular and mandibular RALs with regression during treatment.

\section{Modified RINBO score}

The composite mRINBO score taking the number of RALs, RALmax, extramedullary and spinal lesions into account, decreased from a median of 5 [IQR, 4-7] at baseline to a median of 4 [IQR, 3-5] at year-one, $\mathrm{p}=0.05$ (Fig. 3).

\section{Interrater agreement}

The observer difference regarding the size of RALs was maximum $5 \mathrm{~mm}$ and the mean sizes were therefore used in the estimation of mRINBO. Disagreements regarding the presence of active bone lesions occurred only in relation to two lesions and this discordance was solved by consensus.

\section{Clinical disease activity}

Symptoms were reported in relation to all anatomic RAL subgroups. However, from clinical records it was not always possible to assess if each RAL was symptomatic. At year-one, 8/18 (44\%) children had clinical inactive disease and another $9 / 18(50 \%)$ children reported clinical improvement. In children with clinically inactive disease year one $(n=8)$, the median number of RAL was 3 [IQR, 1-3] pr. patient and the median mRINBO score was 5 [IQR, 2.5-5.5]. In children with clinically active disease year one $(n=10)$, the median number of RAL was 3.5 [IQR, 2-5] per patient and the median mRINBO score was 4.5 [IQR, 3-5]. There was no difference between children with clinically inactive disease and children with clinically active disease at year-one regarding the number of RALs per patient and mRINBO. 


\section{Discussion}

In this study a modified radiological index for non-bacterial osteitis (mRINBO) was used for standardized reporting and quantification of WBMRI findings in children with CNO. mRINBO was assessed initially and after treatment with pamidronate for one year. We implemented modifications of the RINBO-score [21] regarding the size of RALmax and exclusion of RAL in the metatarsal bones.

From baseline to year-one the mRINBO decreased from a median of 5 [IQR, 4-7] to a median of 4 [IQR, 3-5] $(p=0.05)$ driven by a significant decrease of the number of RALs per patient and the size of RALmax whereas the median size of all RALs did not change from baseline to year-one. Signs of reduced radiological activity occurred especially in the pelvis, in the long tubular bones of the lower extremities and in the thoracic spine. Also, signs of extramedullary affection regressed.

When assessing the size of RALmax we observed, that the largest RAL at baseline was $65 \mathrm{~mm}$ and the median size of RALmax was 39 [IQR, 26-45] mm and at year-one, the largest RAL was $78 \mathrm{~mm}$ and the median size of RALmax was 28 [IQR, 20-40] mm. In the original RINBO study the largest RAL was considerable larger being $265 \mathrm{~mm}$ (mean 64 $\pm 55 \mathrm{~mm}$ ).[2] The size of RAL depends on disease activity, but also on the size of the bone involved, implying that the age of the child can influence the size of RAL and RALmax. Therefore, a relative size measure of RAL might be advantageous, but was not obtained in the current study.

Assessment of the metatarsal bone lesions revealed that metatarsal lesions often were clinically silent. Radiological CNO findings in the feet have previously been reported in a WBMRI study of 53 children with $\mathrm{CNO}$. This study also showed a mismatch between radiological and clinical findings in the metatarsal bones.[5] Furthermore, metatarsal bone lesions are difficult to assess by coronal WBMRI and difficult to differentiate from non-specific hyperintensities in the feet which may be normal findings in children.[40] Therefore, if lesions in the forefoot are considered of clinical significance, it is recommended to perform site specific MRI assessment or plain radiographs, which may disclose characteristic CNO changes in the feet, including osteolysis, osteosclerosis and new bone formation. $[9,41]$

An advantage of the mRINBO score assessed is that it follows a systematic quantitative approach and results in a summary patient score. In this study, we assessed the longitudinal changes in radiological disease activity in children with CNO treated with pamidronate. However, mRINBO may also be used to assess radiological changes independently of the therapy used. In clinical practice WBMRI in CNO is often used for diagnosis and not as follow-up,[12] but in clinical studies assessing treatment response a standardized radiological outcome measure is needed.[20] Longitudinal assessment of changes in radiological disease activity in children with $\mathrm{CNO}$ has previously predominantly been descriptive, mainly reporting the number of bone lesions and changes in bone marrow oedema based on expert opinion. $[3,8$, $46,47,29,31,32,36,42-45]$. Changes in radiological disease activity following pamidronate therapy have been reported based on a WBMRI scoring system encompassing the number, relative size and signal intensity of active CNO lesions as well as soft tissue inflammation and structural lesions in the form of osseous hyperostosis and vertebral collapse, but the study was primarily performed to test reliability of 
the MR assessment and did not include global patient scores for disease severity.[23] Furthermore, a consensus-driven method for semiquantitative grading of the various CNO lesions by WBMRI has been described, likewise including both active and structural changes and using the relative size of active bone lesion [22], but this system has to our knowledge not been tested in longitudinal studies probably because further studies are needed to determine the weighting of each variable.

The mRINBO has the advantage of following a systematic quantitative approach reporting the accumulated radiological disease activity in each individual with CNO. Also, the mRINBO quantification is relatively easy to obtain and takes the important features of $\mathrm{CNO}$ into account, considering anatomical locations in addition to lesion number and size. Some anatomical locations may be considered to imply an increased risk such as spinal lesions as they can be osteolytic and lead to vertebral collapse.[7, 8, 48] CNO lesions vary considerably in size; the largest lesions often occurring in the pelvis, and future studies may have to prove that the maximal size of RAL is an important feature of disease activity, measured as absolute or relative value.

Monitoring CNO activity is complicated by the fluctuating disease course with spontaneous development of new RALs, $[3,41]$ also during pamidronate treatment. $[8,36]$ Based on this study, it was not possible to determine if pamidronate prevented formation of new bone lesions.

The improvement regarding the number of spinal RALs was statistically significant, but it was not possible to show improvements in chronic vertebral changes during one year of pamidronate treatment. Previous studies have shown partial reconstitution of vertebral shape in CNO during long lasting pamidronate treatment up to 41 months. [8,27] Also, it is well known that the radiological changes and clinical activity in CNO can resolve spontaneously and independently of treatment.[6] In WBMRI studies, the number of radiological bone lesions have been shown to be lower in cohorts including a mixed population of clinically active and inactive patients $[2,21]$ compared to groups with only clinically active patients. $[3,5]$ Hence, spontaneous improvement of CNO might overestimate the response to pamidronate treatment in this study and controlled trials are warranted.

Further limitations of this study include the performance of WBMRI on two different MRI scanners and the protocol used did not always include a T1-weighted sequence. Systematically performed T1-weighted sequences might have contributed to a higher sensitivity and specificity in assessing bone lesions than STIR alone. Secondly, this study was limited by a small sample size. However, to our knowledge, this is the first study to systematically assess the individual radiological treatment response in children with CNO using a standardized quantification approach. Thirdly, the study population was selected based on inclusion and exclusion criteria regarding $\mathrm{CNO}$ severity, pamidronate treatment and uniform imaging regarding modality and timing. Six children who received TNF-a inhibitor treatment were excluded from the study. Exclusion of these patients might have influenced our results. Exclusion of children not fulfilling the imaging criteria might have led to bias as children not examined by WBMRI might have a milder clinical disease course. Finally, it was not possible retrospectively to assess the exact number of 
clinically active lesions, which may have influenced the lack of correlation between the clinical and radiological response.

\section{Conclusion}

In conclusion, we consider the mRINBO score to be a potential system for standardized reporting and quantification of WBMRI findings in children with CNO. This study also showed, that a standardized WBMRI protocol including coronal STIR and T1-weighted imaging in addition to sagittal imaging of the spine must be recommended to obtain uniform scoring of active and structural radiological changes in $\mathrm{CNO}$. To assure that mRINBO is a feasible and valid scoring tool further studies are needed, including considerations regarding the use of RALmax and relative size measures of active lesions in relation to the size of the bone involved.

\section{Abbreviations}

CNO

chronic non-bacterial osteomyelitis

RINBO

radiological index for non-bacterial osteitis (mRINBO)

mRINBO

modified radiological index for non-bacterial osteitis (mRINBO)

RAL

radiologically active bone lesions

RALmax

maximal size of radiologically active bone lesions

WBMRI

whole-body magnetic resonance imaging

ESR

erythrocyte sedimentation rate

STIR

short tau inversion recovery

NSAIDs

Non-steroidal anti-inflammatory drugs

\section{Declarations}

Ethics approval and consent to participate: The Institutional Review Board approved the study (ref. 201604-2). Approval from the regional ethics committee was applied for but deemed unnecessary according to Danish law.

Consent for publication: Not applicable 
Availability of data and materials: The datasets used and/or analysed during the current study are available from the corresponding author on reasonable request

Competing interests: The authors declare that they have no competing interest

Funding: There was no funding for this project.

Authors' contribution: CMA, AGJ, TH and EMH created the project. CMA and AGJ designed the study. Data was collected by CMA. AGJ and RKF evaluated and scored the WBMRI. AGJ and CMA did the interpretation of data and created the tables, figures and images. CMA and AGJ drafted the manuscript. All authors revised the manuscript for key intellectual content and approved the final manuscript.

Acknowledgements: None

\section{Authors' information:}

C.M. Andreasen, MD PhD, (https://orcid.org/0000-0002-2376-0446)

R.F. Klicman, MD, (https://orcid.org/0000-0001-7356-3582)

T. Herlin, MD, DMSc, professor, (https://orcid.org/0000-0002-6525-3207)

E.M. Hauge, MD PhD, professor, (https://orcid.org/0000-0003-2562-9174)

A.G. Jurik, MD DMSc, professor, (https://orcid.org/0000-0003-2571-0466)

\section{Author affiliations:}

Caroline Marie Andreasen ${ }^{1,3,5}$

Rikke Fuglsang Klicman²

Troels Herlin ${ }^{3,4}$

Ellen-Margrethe Hauge $\mathrm{e}^{1,3}$

Anne Grethe Jurik ${ }^{2,3}$

${ }^{1}$ Aarhus University Hospital, Department of Rheumatology, Aarhus, Denmark.

${ }^{2}$ Aarhus University Hospital, Department of Radiology, Aarhus, Denmark.

${ }^{3}$ Aarhus University, Department of Clinical Medicine, DK-8200 Aarhus, Denmark 
${ }^{4}$ Aarhus University Hospital, Department of Pediatrics and Adolescent Medicine, Aarhus, Denmark.

${ }^{5}$ Vejle Hospital, SLB, Department of Medicine, Rheumatology, Vejle, Denmark

\section{Corresponding author}

Correspondance to: Caroline Marie Andreasen Caroline.Marie.Andreasen@rsyd.dk

\section{References}

1. Ferguson PJ, El-Shanti HI. Autoinflammatory bone disorders. Curr Opin Rheumatol. 2007;19:492-8.

2. Voit AM, Arnoldi AP, Douis H, Bleisteiner F, Jansson MK, Reiser MF, et al. Whole-body magnetic resonance imaging in chronic recurrent multifocal osteomyelitis: Clinical longterm assessment may underestimate activity. J Rheumatol. 2015;42:1455-62.

3. Beck C, Morbach H, Beer M, Stenzel M, Tappe D, Gattenlöhner S, et al. Chronic nonbacterial osteomyelitis in childhood: prospective follow-up during the first year of anti-inflammatory treatment. Arthritis Res Ther. 2010;12:R74.

4. Jurik AG, Klicman RF, Simoni P, Robinson P, Teh J. SAPHO and CRMO: The Value of Imaging. Semin Musculoskelet Radiol. 2018;22:207-24.

5. von Kalle T, Heim N, Hospach T, Langendörfer M, Winkler P, Stuber T. Typical patterns of bone involvement in whole-body MRI of patients with chronic recurrent multifocal osteomyelitis (CRMO). Rofo. 2013;185:655-61.

6. Andreasen CM, Jurik AG, Glerup MB, Høst C, Mahler BT, Hauge E-M, et al. Response to Early-onset Pamidronate Treatment in Chronic Nonbacterial Osteomyelitis: A Retrospective Single-center Study. J Rheumatol. 2019;46:1515-23.

7. Jurik AG. Chronic recurrent multifocal osteomyelitis. Semin Musculoskelet Radiol. 2004;8:243-53.

8. Hospach T, Langendoerfer M, von Kalle T, Maier J, Dannecker GE. Spinal involvement in chronic recurrent multifocal osteomyelitis (CRMO) in childhood and effect of pamidronate. Eur J Pediatr. 2010;169:1105-11.

9. Jansson A, Renner ED, Ramser J, Mayer A, Haban M, Meindl A, et al. Classification of non-bacterial osteitis: Retrospective study of clinical, immunological and genetic aspects in 89 patients. Rheumatology. 2007;46:154-60.

10. Østergaard M, Eshed I, Althoff CE, Poggenborg RP, Diekhoff T, Krabbe S, et al. Whole-body magnetic resonance imaging in inflammatory arthritis: Systematic literature review and first steps toward 
standardization and an omeract scoring system. J Rheumatol. 2017;44:1699-705.

11. Weckbach S. Whole-body MRI for inflammatory arthritis and other multifocal rheumatoid diseases. Semin Musculoskelet Radiol. 2012;16:377-88.

12. Giraudo C, Lecouvet FE, Cotten A, Eshed I, Jans L, Jurik AG, et al. Whole-body magnetic resonance imaging in inflammatory diseases: Where are we now? Results of an International Survey by the European Society of Musculoskeletal Radiology. Eur J Radiol. 2021;136:109533.

13. Fritz J. The Contributions of Whole-body Magnetic Resonance Imaging for the Diagnosis and Management of Chronic Recurrent Multifocal Osteomyelitis. J Rheumatol. 2015;42:1359-60.

14. Guérin-Pfyffer S, Guillaume-Czitrom S, Tammam S, Koné-Paut I. Evaluation of chronic recurrent multifocal osteitis in children by whole-body magnetic resonance imaging. Jt Bone Spine. 2012;79:616-20.

15. Weber U, Lambert RG, Rufibach K, Maksymowych WP, Hodler J, Zejden A, et al. Anterior chest wall inflammation by whole-body magnetic resonance imaging in patients with spondyloarthritis: lack of association between clinical and imaging findings in a cross-sectional study. Arthritis Res Ther. 2012;14:R3.

16. Madsen KB, Jurik AG. MRI grading method for active and chronic spinal changes in spondyloarthritis. Clin Radiol. 2010;65:6-14.

17. Li C, Zhao Y, Zuo Y, Zhou Y, Zhang F, Liu S, et al. Efficacy of bisphosphonates in patients with synovitis, acne, pustulosis, hyperostosis, and osteitis syndrome: a prospective open study. Clin Exp Rheumatol. 2019;37:663-9.

18. Lukas C, Braun J, van der Heijde D, Hermann K-GA, Rudwaleit M, Ostergaard M, et al. Scoring inflammatory activity of the spine by magnetic resonance imaging in ankylosing spondylitis: a multireader experiment. J Rheumatol. 2007;34:862-70.

19. Andreasen CM, Jurik AG, Deleuran BW, Horn HC, Folkmar TB, Herlin T, et al. Pamidronate in chronic non-bacterial osteomyelitis: a randomized, double-blinded, placebo-controlled pilot trial. Scand J Rheumatol. 2020;49:312-22.

20. Jurik AG. Quantification of Disease Activity in Chronic Nonbacterial Osteomyelitis by Whole-body Magnetic Resonance Imaging. J Rheumatol. 2020;47:646-9.

21. Arnoldi AP, Schlett CL, Douis H, Geyer LL, Voit AM, Bleisteiner F, et al. Whole-body MRI in patients with Non-bacterial Osteitis: Radiological findings and correlation with clinical data. Eur Radiol. 2017;27:2391-9.

22. Zhao Y, Sato TS, Nielsen SM, Beer M, Huang M, lyer RS, et al. Development of a Scoring Tool for Chronic Nonbacterial Osteomyelitis Magnetic Resonance Imaging and Evaluation of its Interrater Reliability. J Rheumatol 2019.

23. Panwar J, Tolend M, Lim L, Tse SM, Doria AS, Laxer RM, et al. Whole-Body MRI Quantification for Assessment of Bone Lesions in Chronic Nonbacterial Osteomyelitis Patients Treated With Pamidronate: A Prevalence, Reproducibility, and Responsiveness Study. J Rheumatol 2020:jrheum.200329. 
24. Morbach H, Hedrich CM, Beer M, Girschick HJ. Autoinflammatory bone disorders. Clin Immunol. 2013;147:185-96.

25. Wipff J, Costantino F, Lemelle I, Pajot C, Duquesne A, Lorrot M, et al. A large national cohort of French patients with chronic recurrent multifocal osteitis. Arthritis Rheumatol. 2015;67:1128-37.

26. Taddio A, Ferrara G, Insalaco A, Pardeo M, Gregori M, Finetti M, et al. Dealing with Chronic NonBacterial Osteomyelitis: a practical approach. Pediatr Rheumatol Online J. 2017;15:87.

27. Gleeson H, Wiltshire E, Briody J, Hall J, Chaitow J, Sillence D, et al. Childhood chronic recurrent multifocal osteomyelitis: pamidronate therapy decreases pain and improves vertebral shape. $J$ Rheumatol. 2008;35:707-12.

28. Kerrison C, Davidson JE, Cleary AG, Beresford MW. Pamidronate in the treatment of childhood SAPHO syndrome. Rheumatology. 2004;43:1246-51.

29. Miettunen PMH, Wei X, Kaura D, Reslan WA, Aguirre AN, Kellner JD. Dramatic pain relief and resolution of bone inflammation following pamidronate in 9 pediatric patients with persistent chronic recurrent multifocal osteomyelitis (CRMO). Pediatr Rheumatol. 2009;7:1-14.

30. Roderick MR, Shah R, Rogers V, Finn A, Ramanan AV. Chronic recurrent multifocal osteomyelitis (CRMO) - advancing the diagnosis. Pediatr Rheumatol Online J. 2016;14:47.

31. Simm PJ, Allen RC, Zacharin MR. Bisphosphonate treatment in chronic recurrent multifocal osteomyelitis. J Pediatr. 2008;152:571-5.

32. Schnabel A, Range U, Hahn G, Berner R, Hedrich CM. Treatment Response and Longterm Outcomes in Children with Chronic Nonbacterial Osteomyelitis. J Rheumatol. 2017;44:1058-65.

33. Pastore S, Ferrara G, Monasta L, Meini A, Cattalini M, Martino S, et al. Chronic nonbacterial osteomyelitis may be associated with renal disease and bisphosphonates are a good option for the majority of patients. Acta Paediatr Int J Paediatr. 2016;105:e328-33.

34. Al Hajry M, Al Jumaah S, Almayouf SM. Chronic recurrent multifocal osteomyelitis: a first report from Saudi Arabia. Ann Saudi Med. 2012;32:611-4.

35. Moussa T, Bhat V, Kini V, Fathalla BM. Clinical and genetic association, radiological findings and response to biological therapy in seven children from Qatar with non-bacterial osteomyelitis. Int $J$ Rheum Dis. 2017;20:1286-96.

36. Roderick M, Shah R, Finn A, Ramanan AV. Efficacy of pamidronate therapy in children with chronic non-bacterial osteitis: disease activity assessment by whole body magnetic resonance imaging. Rheumatology. 2014;53:1973-6.

37. Nixon GW. Hematogenous osteomyelitis of metaphyseal-equivalent locations. AJR Am J Roentgenol. 1978;130:123-9.

38. Laor T, Jaramillo D. Metaphyseal abnormalities in children: pathophysiology and radiologic appearance. AJR Am J Roentgenol. 1993;161:1029-36.

39. Ponseti IV. Growth and development of the acetabulum in the normal child. Anatomical, histological, and roentgenographic studies. J Bone Joint Surg Am. 1978;60:575-85. 
40. Shabshin N, Schweitzer ME, Morrison WB, Carrino JA, Keller MS, Grissom LE. High-signal T2 changes of the bone marrow of the foot and ankle in children: red marrow or traumatic changes? Pediatr Radiol. 2006;36:670-6.

41. Jurik AG, Helmig O, Ternowitz T, Møller BN. Chronic recurrent multifocal osteomyelitis: a follow-up study. J Pediatr Orthop. 1988;8:49-58.

42. Hofmann C, Wurm M, Schwarz T, Neubauer H, Beer M, Girschick H, et al. A standardized clinical and radiological follow-up of patients with chronic non-bacterial osteomyelitis treated with pamidronate. Clin Exp Rheumatol. 2014;32:604-9.

43. Eisenstein EM, Syverson GD, Vora SS, Williams CB. Combination therapy with methotrexate and etanercept for refractory chronic recurrent multifocal osteomyelitis. J Rheumatol. 2011;38:782-3.

44. Borzutzky A, Stern S, Reiff A, Zurakowski D, Steinberg EA, Dedeoglu F, et al. Pediatric chronic nonbacterial osteomyelitis. Pediatrics. 2012;130:e1190-7.

45. Batu ED, Ergen FB, Gulhan B, Topaloglu R, Aydingoz U, Ozen S. Etanercept treatment in five cases of refractory chronic recurrent multifocal osteomyelitis (CRMO). Jt Bone Spine. 2015;82:471-3.

46. Zhao Y, Chauvin NA, Jaramillo D, Burnham JM. Aggressive Therapy Reduces Disease Activity without Skeletal Damage Progression in Chronic Nonbacterial Osteomyelitis. J Rheumatol. 2015;42:124551.

47. Tronconi E, Miniaci A, Baldazzi M, Greco L, Pession A. Biologic treatment for chronic recurrent multifocal osteomyelitis: report of four cases and review of the literature. Rheumatol Int. 2018;38:153-60.

48. Schultz C, Holterhus PM, Seidel A, Jonas S, Barthel M, Kruse K, et al. Chronic recurrent multifocal osteomyelitis in children. Pediatr Infect Dis J. 1999;18:1008-13.

\section{Figures}

\section{Figure 1}

\section{Flowchart.}

Fifty-one children were diagnosed with chronic non-bacterial osteomyelitis (CNO) according to the Bristol Criteria. Children with symmetrical multifocal or spinal inflammatory bone lesions and a poor clinical response to non-steroidal anti-inflammatory drugs were treated with pamidronate $(n=32)$. Reasons for study exclusion were insufficient imaging or concomitant treatment with TNF-a inhibitors. Eighteen children were included in the study. WBMRI: whole-body magnetic resonance imaging 

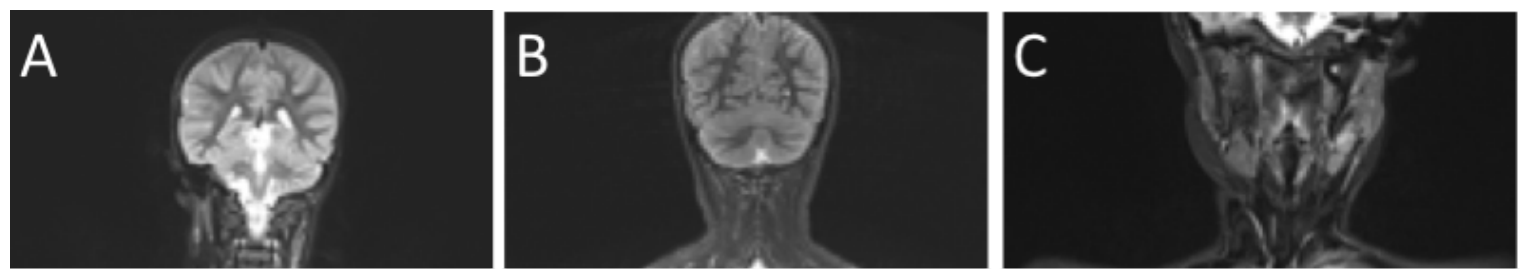

\section{Figure 2}

Whole-body MRI following one year of pamidronate treatment

Representative MRI images of a 7-year-old girl diagnosed with chronic non-bacterial osteomyelitis (CNO) and imaging following one year of pamidronate treatment. (A) Pre-pamidronate whole-body magnetic resonance imaging (WBMRI) short tau inversion recovery (STIR), coronal image shows multiple areas of bone marrow edema as signs of radiologically active lesions (RAL) (white thin arrows). (B) Post- 
pamidronate coronal WBMRI STIR image showed resolution of RAL in the left acetabulum and regression of RAL in the right femur. Assessment of RAL in the distal femur and proximal tibia is challenged by bisphosphonate bands (dotted arrows) (C) Pre-pamidronate and (D) post-pamidronate coronal MRI STIR images of the clavicles showing post-treatment regression of bone marrow edema and surrounding soft tissue edema.

\section{Figure 3}

Changes in radiological disease activity following one year of pamidronate treatment

Radiological response to pamidronate treatment in children diagnosed with severe CNO $(n=18)$. WBMRI was assessed prior to pamidronate treatment and following one year of treatment. (A) Changes in the modified radiological index for non-bacterial osteitis (mRINBO), $p=0.05$ (B) Changes in total number of radiologically active lesions (RAL) per patient, $p=0.02$ (C) Changes in the size of the largest RAL for each patient (RALmax) $p<0.01$. (D) Changes in the number of RAL for each patient in the spine, $p<0.01$. Comparison between baseline and year one was made using Wilcoxon signed rank test.

\section{Supplementary Files}

This is a list of supplementary files associated with this preprint. Click to download.

- 22.01.05Supplementarymaterial.docx 